\title{
Synergetic Recollection: How to Design Lifelogging Tools that Help Locate the Right Information
}

\author{
Vaiva Kalnikaitè \\ Interactables \\ 23 Kings Street, Cambridge \\ CB1 1AH, UK \\ vaiva@interactables.com
}

\author{
Steve Whittaker \\ Psychology Department \\ University of California at Santa Cruz \\ CA95064 \\ swhittak@ucsc.edu
}

\begin{abstract}
Recent lifelogging research has been primarily focused on developing new technology to support the capture of everyday events and experiences. Less attention has been spent on understanding what types of lifelogging tools have been built so far and how these different tools help reconstruct memories of the past. In this chapter, we discuss empirical research showing which lifelogging practices and features can help recollection. We explore psychology research for effective memory cuing such as the use of associations with other people, and we discuss the synergies between organic and synthetic recall, driven by the selfaware process of remembering - also known as metamemory. Finally, we conclude this chapter by discussing the impact of metamemory on personal remembering and reflect on principles for the design of future lifelogging tools.
\end{abstract}

\section{Introduction}

Organic memory is crucial in everyday remembering, and the fallibility of organic remembering is well understood (Schacter 1987; Schacter 1997; Baddeley 1998; Anderson 2004). With the proliferation of mobile and ubiquitous technologies, it is now possible to imagine capturing vast amounts of personal information about our past on an external device in digital form. An optimistic vision of this practice is described in a recent book by Gordon Bell and Jim Gemmell, titled "Total Recall” (Bell and Gemmell 2009). We refer to this activity of mass recording and storage of personal experience as synthetic remembering, or lifelogging. Synthetic memory is not a hypothetical tool: our daily lives are already full of todos and shopping lists, diaries and post-it notes, as well as smart phones and wearable sensing devices that share a common purpose: to capture, store and allow us to retrieve things we want to remember but would otherwise forget.

Lifelogging research has so far focused on technical innovation rather than understanding when, why or how these tools are used in practice. Similarly, psychology research on memory primarily focuses on understanding organic memory functions in isolation. But in practice, in our current everyday lives, we seldom rely solely on our organic memory to remember. For example, we don't expect to be able to recall, simply from memory, the contact details of every person we meet at a conference. Our natural reaction is to store this information somewhere on an external lifelogging device - whether a business card in a wallet, or an entry in a phonebook - and then retrieve it when needed. Effectively capturing relevant information is an important part of this process, and several research efforts have focused on creating tools that can exhaustively capture and log daily experience, such as MyLifeBits (Gemmel 2006), Haystack (Karger 2004) or Stuff I've Seen (Dumais, Cutrell et al. 2003). While these projects have contributed significant advances in capturing and cataloging data, 
they have paid less attention to exploring how, when and why people retrieve events and information from these large personal archives. Techniques for effective recall are crucial for guiding memory processing to a piece of information - this is the basis of the process of remembering. But it is not enough to be able to access some information - once a memory is retrieved, it is essential to be able to assess whether it contains the right information. This ability is part of a process known as metamemory, which is fundamental part of the memory recall process. And yet, although there is extensive psychology research in understanding the function and role of metamemory, this knowledge is rarely taken into account when designing new lifelogging technologies.

The concept of metamemory was introduced by Lachman, Lachman and Thronesberry (Lachman, Lachman et al. 1979) and has been widely applied in psychology research to help understand and explain the underlying processes that drive organic remembering. It is an active process of motivated reassurance that guides the remembered information through the right channels of validation. The question is: do we apply the same validation and guidance processes when retrieving information from a lifelog? We frequently treat lifelogs as a synthetic extension or prosthesis of our organic memory, where we can archive larger amounts of information beyond the capacity of our organic memories. Despite being able to make use of sophisticated information repositories, which support flexible storage, indexing, clustering, other tools for organization and retrieval, picking out memories of past events from these vast repositories of personal information still remains a challenge. There is also very limited research in understanding how we can effectively cue these repositories - how do we provide effective indexes or clues that allows to quickly identify important information. There have been multiple attempts to cluster and re-organise this personal information through the use of temporal cues, such as the SenseCam Diary (Doherty 2009), but previous psychology research suggests that people are not able to reliably remember specific times (Cohen 1996).

This chapter bridges between organic and synthetic remembering by exploring the following research questions in detail:

1) How does metamemory support organic remembering?

2) How do different types of lifelogging tools help cue memory?

3) When and why do people rely on organic remembering as opposed on synthetic remembering?

4) How does metamemory influence and explain lifelogging practices?

5) What should be considered when designing future lifelogging tools?

\section{Self-aware Organic Remembering - Metamemory Function}

According to Schacter, there is no universally agreed model of the mind (Schacter 1997) and there is no agreement on how memory works overall. Some memory models are based on cognitive psychology, with an assumption that the brain operates like a computer (Johnson 
Laird 1988), but Schacter disagrees, arguing that these models cannot account for the so called objective, present-need and just-in-time basis of memory. Subjectivity in remembering, Schacter says, involves at least three important factors: 1) memories are constructions made in accordance with present needs, desires, influences, and other factors; 2) memories are often accompanied by feelings and emotions; and 3) memory usually involves the rememberer's awareness of the memory - metamemory.

According to Schacter, a good model of how memory works must not only fit with specific knowledge but also with the subjective nature of memory itself. Memory debates amongst psychologists have resulted in different theories and explanations of how memories might be produced, stored and reconstructed. However, many of these explorations have been conducted in a laboratory setting, or based on a study of small numbers of brain-damaged patients. Even fewer studies have taken a naturalistic approach, where participants are asked to remember every day and personal materials such as conversational stories, films or personal events (Linton 1982; Cohen, Kiss et al. 1993).

Despite being able to understand how exactly memory works as a whole, there has been much research into understanding what could be considered as effective memory cues (Cohen 1993; Cohen et al 1996). Generally, we are good at predicting those things that we will be able to remember, and know what things we are likely to forget. This ability to 'know what we know' is referred to as metamemory. Lachman et al demonstrated that metamemory is generally accurate (i.e. people correctly predict what they will remember), and that most people can direct their memory search effectively and productively. These results prove that people really do 'know their own memories'. According to (Cavanaugh 1988) there are three types of metamemory processes: systemic awareness, epistemic awareness and on-line awareness. These three types of metamemory are interrelated. Systemic awareness consists of knowing what kinds of encoding and retrieval produces the best results, as well as what kind of things are difficult or easy to remember. For instance, it is hard to memorise long words that do not have any meaning. Epistemic awareness consists of knowing what we know and being able to make judgments about the accuracy of what we know. For instance, knowing that there is a double "b" and a double "o" in the word "gobbledegook" and being able to apply grammar rules to determine the accurate location of those double letters. On-line awareness consists of knowing about ongoing memory processes and being able to monitor memory functions, such as whether one is correctly remembering someone's name when one sees them. Thus, when trying to recall a particular fact, epistemic awareness will be needed to identify what relevant information is potentially accessible. Systemic awareness may guide the selection of search strategies directing the search, and on-line awareness might be involved in keeping track of progress during the search. To sum up, Cavanaugh suggested that metamemory refers to the knowledge of how we remember (systemic awareness), the process of monitoring out memory processing (epistemic awareness) and the beliefs we hold about memory (on-line awareness).

Online awareness of metamemory allows us to make a decision about the accuracy of the memory as it is being retrieved. Research investigating the relationship between confidence and accuracy in metamemory has been carried out (Brewer and Sampaio 2006) which shows that metamemory confidence in recently completed memory performances plays a major role in the use of memory in everyday mental activities. The way that metamemory helps is by guiding memory searches, e.g. if one has misplaced ones keys, metamemory will help the memory by guiding it through the finding process (e.g. when did you last see the keys? Can 
you visualise where they were? Is this definitely the last place where you saw them? etc). Furthermore, Brewer and Sampaio claim that whenever individuals have to act on a memory operation that has just been completed, the use of those memories is mediated by their confidence in the accuracy of the memory product. These findings are crucial in our justification for the concept of Lifelogging. Appropriate Lifelogging tools can influence perceived accuracy, increasing user confidence that synthetically retrieved memories are accurate regardless of the confidence state when remembering organically. They also motivate the initial use of a lifelogging tool. If someone is aware that particular information is likely to be forgotten then they are more likely to take strategic action to remember e.g. by carrying a lifelogging tool. Similarly, at retrieval, if people are aware of the shortcomings of their organic memory then they might be more likely to rely on an external record for accurate retrieval. Other studies looked into models that could explain how metamemory processes are used to regulate memory accuracy and quantity performance under free-report conditions (Koriat, Goldsmith et al. 2000). According to the model, when recounting past events, people monitor the likelihood that each item of information that comes to mind is correct. They then apply a control threshold to the monitoring output for the item with the highest subjective probability of being correct. The item will be reported if its assessed probability passes the threshold, and will be withheld otherwise. The control threshold is set on the basis of implicit or explicit payoff. In other words, the relative utility of providing complete versus accurate information. The stronger the motivations for accuracy, the more selective people are in their reporting the higher level of accuracy is attained. But the drawback of this is that individuals can achieve a high level of memory accuracy by withholding a large number of correct answers.

There are a number of phenomena associated with metamemory. The Tip of the Tongue (TOT) phenomenon involves epistemic metamemory as shown in studies by Brown (Brown 1991). Feeling of Knowing (FOK) (Wellman 1977) is distinguished from the TOT state, as relating to a whole range of knowledge states: from being sure that one does not know something, to being confident that one can recall it (if given enough time and/or suitable cues).

It is obvious that metamemory directly applies to lifelogging practices and could help explain how, when and why people Lifelog. Metamemory determines whether memories have been forgotten or not. In the case when memories are forgotten, the decision to use lifelogging tools might be prompted by metamemory processes. However, this raises further research questions around how people retrieve forgotten memories from their organic memories, or decide to rely on Lifelogs for retrieval. Metamemory is a central process that involves awareness of when we forget, as well as about the efficiency of different memory strategies and cuing processes. It is crucial for the study of lifelogging which involves an intentional deployment of tools to meet perceived memory goals. A better understanding of metamemory should inform better understand of how and when lifelogging tools are used. Finally, it helps determine how they should be designed for working in synergy with organic memory.

\section{Synergetic Remembering - Lifelogging Vision and Practices}

We now know that organic memory is fallible, and this leads to active preparation for recall through lifelogging. Generally lifelogging could be defined as a constellation of tools that extend the capacity of organic memory that have the potential to capture important 
mnemonic information. Lifelogging tools frequently use passive capture - without active intervention from the user, often engaging sensor triggers. But on occasions, lifelogging could be active - where users engage with the tool via an activity e.g. press a button to take a picture or record a sound snippet. These tools facilitate non-deliberate capture of everyday events. With passive capture in particular, people do not have to make a decision as to what is being captured, e.g. they put on a wearable piece of technology that captures relevant memory data when automatic triggers become actively engaged. However, the predominant questions for either of these forms of capture are: how well do these capture tools support organic memory? With a few exceptions (Whittaker, Hyland et al. 1994; Sellen, Fogg et al. 2007; Harper, Randall et al. 2008, Kalnikaite et al 2010) there has been little work on systematically evaluating these tools.

Lifelogging is often unintentional and eliminates the process of any conscious decision making over when and what to capture. Some examples of lifelogging tools are: SenseCam (Hodges, Williams et al. 2006; Sellen, Fogg et al. 2007), StartleCam (Healey and Picard 1998) and Video Summary System (Aizawa, Tancharoen et al. 2004) which are wearable cameras that take pictures at frequent intervals. Lifelogging tools often capture the surrounding environment as well as any interactions with other people and objects. More often than not, the actual Lifelogger gets excluded from the information logs. Sometimes lifelogging tools can infer the presence of the Lifelogger by displaying familiar things or a familiar environment. Lifeloggers are less likely to pose or be able to create active self-reference. So an active Lifelogger generally prepares for recalling the world as an observer.

With the growth of various forms of technology, in particular cost-effective storage, we have seen a proliferation of lifelogging devices. Many innovative lifelogging tools have emerged in the past few decades. Since Bush's Memex vision (Bush 1945) there has been much interest in building prosthetic tools that are designed to aid organic memory. Based on the context and types of recollections that these Lifelogs support, lifelogging tools could be segmented into two overarching categories:

"Things I Did" - these are events that people actively took part in, e.g. lifelogging while travelling or attending an interesting lecture. The tools that provide support for these types of Lifelogs focus primarily on recording events that are mobile: a) Mobile Capture; or are specific to a particular domain, e.g. capturing in specific meetings or lectures so called: b) Domain Specific Capture.

"Stuff I Have" - is concerned with all the information that we come in touch with e.g. downloading or collecting or otherwise accumulating through the continuous use of tools e.g. e-mail. This category is oriented towards the tools that facilitate organised archiving of digital items: Infrastructure Repositories;

"Things I did" generally encompasses autobiographical events in people's lives that are of important and personal nature. Often these are special events or occasions that people may have already recorded on their digital cameras or other lifelogging tools, e.g. weddings, graduations, birthdays, holidays. More complex lifelogging tools allow people to capture and synchronise multiple multimedia objects e.g. combining audio, images, video, notes, augmented physical objects and sensor data to make a rich meeting record. Many of these event capture tools tend to be in the domain of Mobile Capture - away from the desktop and often when users are on the move. Domain Specific Capture is also focused on events, but usually in a specialised domain area: e.g. speech in meetings, lecture streams or any other 
form of communication requiring a mnemonic record of knowledge. "Stuff I have" in contrast encompasses an Infrastructure Repository for storing collections of heterogeneous digital items that users encounter and archive, including web pages, documents, e-mails, voice-mail and photos. These clusters are described below and illustrated in Figure 2.

\subsection{Overview of "Things I Did"'Tools}

\section{Mobile Capture}

With the proliferation of lifelogging tools, there has been much emphasis on mnemonic visual capture with wearable digital cameras and other wearable devices in a mobile context. These devices are so pervasive that they often get worn and forgotten by the users. Mobile capture also focuses on augmenting physical memorabilia with digital narrative and associations.

\section{Mnemonic Visual Capture}

SenseCam (Hodges, Williams et al. 2006) is a wearable lifelogging device that allows either sensor based or temporal based automated image capture. Images are taken when there are changes in light intensity, posture, or after a specified time interval. From user evaluations it was evident that passively captured images with SenseCam were an effective cue for helping to retrieve past events, when compared to active capture, which was less effective (Sellen, Fogg et al. 2007). SenseCam has also been a significant memory aid for patients suffering from various memory deficiencies (Berry, Kapur et al. 2006). In addition SenseCam supports detailed recall when compared to recalling with just locational information (Kalnikaite, Sellen et al. 2010).

StartleCam is a predecessor to SenseCam containing a skin conductivity sensor trigger, which is sampled by an analogue to digital converter attached to a wearable computer. Images are captured by a permanently connected camera. When the computer detects a response in the user, i.e. a change in skin conductivity, the buffer of images is transmitted wirelessly over the Internet to a remote server. The user may then view the recordings to trigger their memory. The startle detection filter was demonstrated to work with a few wearers (Healey and Picard 1998).

Steve Manns's EyeTap is another personal experience capturing system - where the user wears digital eyeglasses that cause the eye to function as if it were both a camera and a display. EyeTap captures videos as they were originally seen by the user. It provides continuous visual Lifelog which can be shared with others. There are clear benefits in using these technologies for surveillance and archiving personal life, but there are no user studies investigating these effects (Mann, Fung et al. 2005).

In addition to mnemonic video capture, Memory Glasses (DeVaul, Pentland et al. 2003) aims to provide just-in-time memory support. This tool uses the wearer's context as a relevant reminder of the situation and sends the user low-attention subliminal cues in the form of images or text. User studies have shown that effective support for organic memory requires situation-appropriate information, which was provided by Memory Glasses in this case.

eyeBlog (Dickie, Vertegaal et al. 2004) is another automatic personal video recording and publishing system. A wearable glasses camera captures videos that are uploaded in a chronologically delineated blog. This tool can capture face-to-face communications as well 
as record other visuals based on some pre-set pattern, e.g. glyphs. This allows users to control what is automatically captured, but also edit it later once it's been uploaded onto the blog.

\section{Mnemonic Memento-Event Based Capture}

Ubiquitous Memories (Kawamura, Fukuhara et al. 2007) is a human memory augmentation system that enables users to associate experience data with a physical object. User studies have shown that this tool supports memorisation and recollection of past events.

The Living Memory Box (Stevens, Abowd et al. 2003) multimedia appliance can be seen as both an archival and narration device, allowing families to bring artifacts and tell stories about these mementos. This tool captures stills, video and audio about physical objects placed in the box and gathers metadata about them, e.g. date, time and place. User feedback about the initial prototype was positive; however users felt they wanted to be able to annotate their own mementos instead of having automatic annotation. They wanted to be encouraged by the system to tell stories about their mementos and they also wanted to be able to include multiple voices in their speech-based narratives.

Frohlich and Murphy's Memory Box is a wooden box that contains physical mementos. When these mementos are removed from the box, an individual story or an ambient sound associated with that memento is played back automatically. Overall, users responded positively to the idea of attaching stories to the souvenirs, as long as this process was simple and was part of how these objects were handled (Frohlich and Murphy 2000).

Finally, Petrelli and Villar et al's (Petrelli and Villar et al. 2010) FMRadio allowed people to audio record their family holidays and upload them into predefined channels on a physical radio. Families were able to recollect and reminisce about their family holiday months later using this novel, but yet very familiar device. Ambient and other sounds of voices evoked detailed recollection as well as reminiscence.

\section{Domain Specific Capture}

This type of intentional capture technology focuses on recording domain specific information, e.g. meetings, lectures, and other domains where there is a great demand on organic remembering emphasising on verbatim recall.

\section{Meeting Capture}

Great memory demands are present in specific domains. Thus people often use PM tools for these situations. However despite extensive development of "meeting capture" tools, people still tend to rely on simple pen and paper to capture important information in meetings. A few of these tools are described below and illustrated in Figure 1.

Whittaker et al.'s Filochat system combines an audio recorder with a tablet PC for taking notes as a means of constructing a meeting record. Notes are temporally co- indexed with audio, so that clicking on a note accesses the speech that occurred when the note was taken. The tablet PC provides a notebook base that allows users to store several pages of notes and organise them into sections. Users can then use the notes they have taken to jump to the 
relevant portion of the conversation. The system proved successful both in field and lab experiments (Whittaker, Hyland et al. 1994).

Another audio-based lifelogging tool - NotePals is a lightweight note sharing system that gives users easy access to each other's experiences through their personal notes developed by Davis el al (Davis, Landay et al. 1999). The system allows notes taken by users in any context to be uploaded to a shared repository. Users view these notes with browsers that allow them to retrieve all the notes taken in a given context or to access notes from other related meetings or documents. This is possible because NotePals records the context in which each note is created (e.g., its author, subject, and creation time). The system is "lightweight" because it fits easily into group members' regular note-taking practices, and uses informal, ink-based user interfaces that run on portable hardware. This PM proved to be successful for sharing notes between users.

Stifelman's et al.'s Audio Notebook is another audio based tool combining the familiarity of taking notes on physical paper and pen with the advantages of an audio recording. This device augments an ordinary paper notebook, synchronising the user's handwritten notes with a digital audio recording. As with Filochat (Whittaker et al.), the user's natural activities of writing and page turning, implicitly indexes into audio. Users can explore their notes using spatial layout or use time-based navigation to access the audio recordings. Small field studies showed that the interaction techniques enabled a range of usage styles, from detailed review to high speed skimming of the audio (Stifelman, Arons et al. 2001). However there was no objective evaluation of how well this system helped with information retrieval.

Hayes et al.'s Personal Audio Loop (PAL) is another audio based continuous, near-term audio buffering system running on a mobile phone (Hayes, Patel et al. 2004). Audio is constantly buffered but then deleted unless user decides they want to keep it. However from the lab study and diary studies users said that they would like to use this tool more and they liked its compatibility with a mobile phone and its portability. However, study results did not provide any objective benefits of using this tool to aid organic remembering.

Another tool - Dynomite (Wilcox, Schilit et al. 1997) is a pen-based system that allowed users to take notes associated with meetings. A critical feature of the system was that users could classify their notes into different types (e.g. 'todo', phone number, name, date, URL, etc.), which allowed users to create different views of their notes (e.g. all my notes about 'todos' for the last month). Note-taking behaviour was also used to control audio recordings so that although all audio was recorded, only portions that were accompanied by note-taking activity were saved - on the grounds that these were more likely to be of importance. There were no controlled lab or field user evaluations carried out with this system.

Vemuri et al.'s iRemember is an audio based wearable system (Vemuri, Schmandt et al. 2006). This system facilitates capture of life experiences in a variety of everyday situations. This PDA based tool captures and transcribes audio in real time. The transcribed information is presented to the user in a searchable interface. However user evaluation was limited to one author recording everyday conversations with colleagues for 2 years. This limited results of this user evaluation suggested a preference for visual memory to trigger search.

There are also commercial products built to capture meetings in a professional capacity. Quindi is one such tool (Rosenschein 2004). This PC based application captures: audio; video; presentation slides; and, typed notes - fully indexed and ready for search and navigation. Since this is a commercial product, there are no published user evaluations. 
Other research meeting systems include AMI's speech browsers (Tucker and Whittaker 2006) and speech access and processing techniques developed at the University of Toronto (Munteanu, Penn et al. 2006; Munteanu, Baecker et al. 2008) and numerous speech recognition engines developed at Carnegie Mellon University (2009).

\section{Information Capture for Learning}

Another area that places a great demand on memory is the area of learning. Theories of learning are too specialised to be included in this chapter; instead our focus is on lifelogging tools that can support organic remembering where the demand on memory is particularly high - in a learning environment.

Brotherton et al's eClass (Brotherton and Abowd 2004) supports multimedia capture and supplements the regular learning experience with digital video recordings of lectures, slides, digital whiteboard activity, and personal digital notes. User evaluation surveys showed students felt that access to the eClass recordings allowed them to participate more effectively in classes. More quantitative learning benefits were not so clear however. eClass users performed no better on assignments than those using regular teaching materials.

Similar learning tools include Ratto et al.'s Active Class (Ratto, Shapiro et al. 2003), Berque et al.'s Debbie (Berque, Johnson et al. 2001), Anderson et al.'s Classroom Presenter (Anderson, Anderson et al. 2004; Wilkerson, Griswold et al. 2005), and Berque et al.'s DyKnow (Berque, Bonebright et al. 2004). A more traditional approach supporting handwritten annotations, Harvel et al.'s NoteNexus (Harvel, Scheibe et al. 2005) and Kalnikaité and Whittaker's ChattyWeb (Kalnikaite and Whittaker 2010) showed increased access for materials directly related to assessments. Similarly, increased response for materials related to assessment was observed in tools that supporting pictorial annotations: Kalnikaite and Whittaker's PiccyWeb (Kalnikaite and Whittaker 2010). Tools that support social summarization and Web 2.0 in memory demanding set-ups are particularly effective (Kalnikaite and Whittaker 2008).

\subsection{Overview of "Stuff I Have"'Tools}

\section{Infrastructure Repositories}

Tools that store Infrastructure Repository data focus more on providing a knowledge base of significant digital or physical items of information. These tools act more as a complementary reference to underlying life events rather than the actual record of those events. People can just tap into them as and when required for constantly re-occurring memory tasks. There are numerous lifelogging tools that support this type of remembering and archiving. A few of the key tools are outlined below, and illustrated in Figure 1.

MyLifeBits (Gemmell, Bell et al. 2006) is a 'personal database for everything', which is intended to store all information. It focuses on archiving any form of communication or digital memento, e.g. office documents, email, digital photos or scanned images, cardiovascular readings. The tool also allows people to store context information, e.g. GPS import and map displays. It also stores other information that might be relevant to users, e.g. radio capture, TV capture, telephone capture, as well as "flashbulb" news extracts. All stored data is presented to the user in a temporally ordered archive.

Karger and Quan's Haystack (Karger and Quan 2004) is a general-purpose information management environment designed to integrate multiple data sources. It treats any data 
collection the same, regardless whether it is a bookmark hierarchy, a photo album or a mail. User studies in Haystack suggested that users benefited significantly from this added crossdomain flexibility.

Dumais et al.'s Stuff I've Seen (Dumais, Cutrell et al. 2003) (SIS) is a tool facilitating information re-use. The system provides a unified index of all information that a person has seen, whether it was an e-mail, web page, document or other. The system also creates rich contextual cues for encountered information that can be used when retrieving this information. SIS has been thoroughly evaluated with the users, where recent data and people related data were found to be the most accessed.

Presto (Dourish, Edwards et al. 1999) is another document management system which, instead of organising documents in a traditional hierarchy, provides interaction through several document attributes, such as "word file", "published paper", "shared with Jim" or "currently in progress". These are similar to the tags applied in many Web 2.0 systems such as Flickr, del.ici.ous and CiteUlike. These attributes capture different roles that a document might have, which allows users to rapidly re-organise their documents based on the task at hand.

Pblat (Cutrell, Robbins et al. 2006) is a desktop document annotation and location tool. It attempts to integrate both search and browsing functionality through a variety of associative and contextual cues. Phlat supports a unified tagging scheme for organising personal content across various storage systems and types of files, e.g. documents, e-mails and appointments. Phlat has been thoroughly evaluated, with results showing that user generated metadata becomes increasingly critical for how users organise their content. Tagging was preferred over filing in the longer term.

Remembrance Agent (Rhodes 2000) is a contextually aware associative memory tool for just-intime information capture from the web. It automatically caches web pages as they are loaded, adding hyperlinks to personal files. It pre-indexes e-mail archives, documents, and other files based on keyword co-occurrence. User feedback after using Margin Notes was generally positive.

Forget-me-not (Lamming and Flynn 1994) is a system that collects information about selected aspects of the user's activities and organises this data into personal biographies. It can dynamically identify location and interactions with other users, which get automatically logged in the system. It can later prompt users about their activities. However, due to a complex infrastructure set up, user testing was limited to one specific location.

Rekimoto's TimeScape (Rekimoto 1999) is a tool for spatially arranging information on the desktop. It supports "time-travel" to the past or the future of the desktop. The ability to spatially arrange information and chronologically navigate through it allows users to archive electronic information without having to worry about document folders or other file classifications.

Finally, Kalnikaite and Whittaker's MemoryLane (Kalnikaite and Whittaker 2011) is a tool that allows people to manage, organise and interact with long and short-term digital representations of personal physical mementos in the context of people, places and objects. The purpose of the tool is to support autobiographic recollection of past events associated with mementos in MemoryLane and organize them based on ever changing social interactions. 


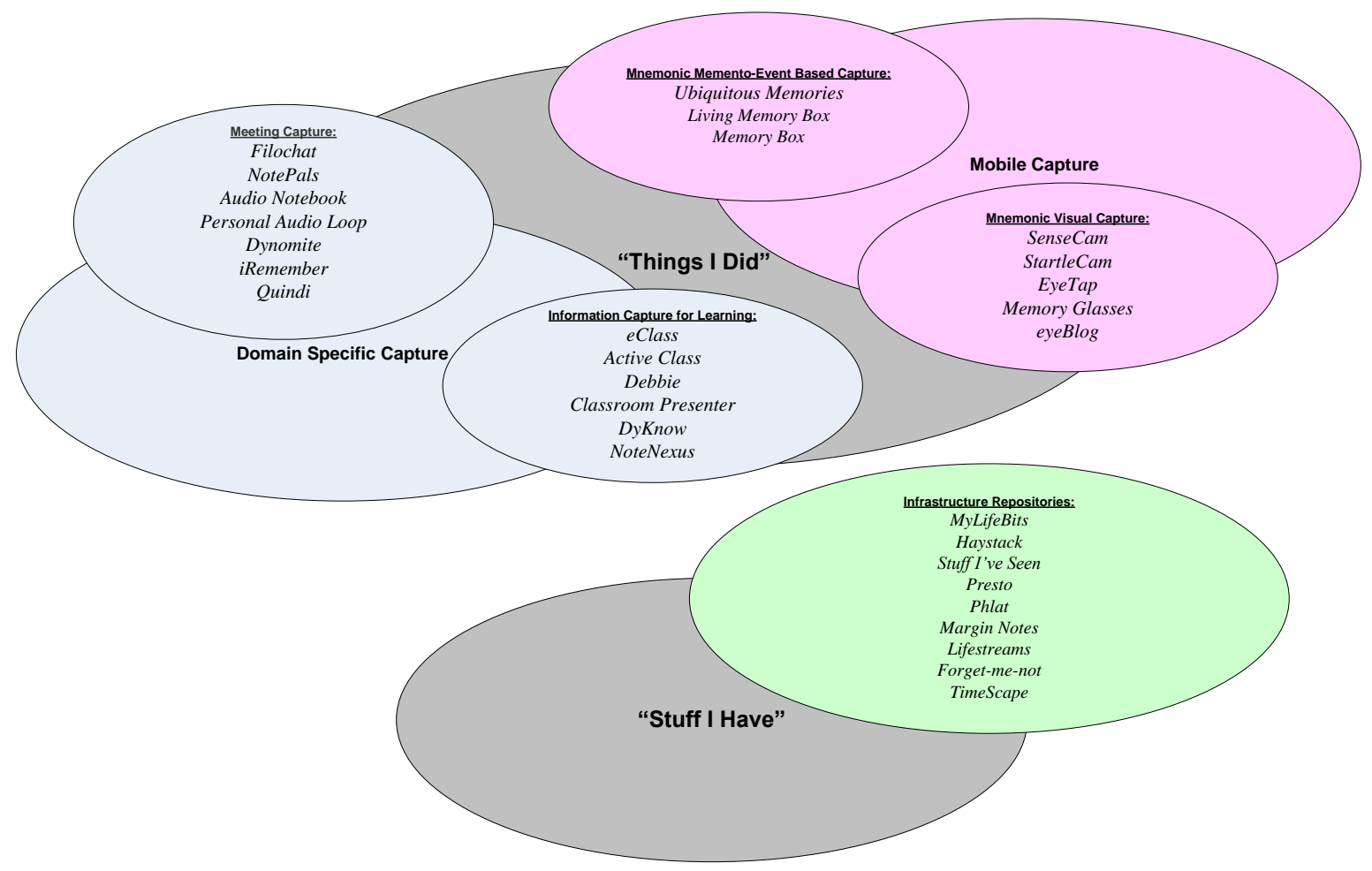

Figure 1: Lifelogging tool segmentation and examples.

\section{Synergetic Remembering - Metamemory Vision}

Now let us consider the case when people not only have access to organic memory, but also to a Lifelog, simultaneously. Figure 2 illustrates a scenario where a person receives a demand for memory. This memory might be archived in the repository of organic memory or a Lifelog. The first thing that metamemory does is evaluate whether this information can be retrieved from organic memory (assuming the preference for organic remembering, as it is usually more efficient). Efficiency is an important determinant for Lifelog use at retrieval. Lifelogging tools that store vast amounts of data might be very inefficient and tedious to use. Thus, if this item of information cannot be retrieved from organic memory alone, metamemory decides in which part of Lifelog it might reside. After interrogating, browsing or otherwise searching through the Lifelog, and finally finding same trace of the information required, metamemory then evaluates whether the memory in question is the actual memory that needs to be remembered. This millisecond process can be repeated multiple times until the point of successful retrieval is reached or the search gets abandoned. Metamemory takes the role of the intermediate agent constantly evaluating and communicating between organic memory and a Lifelog. 


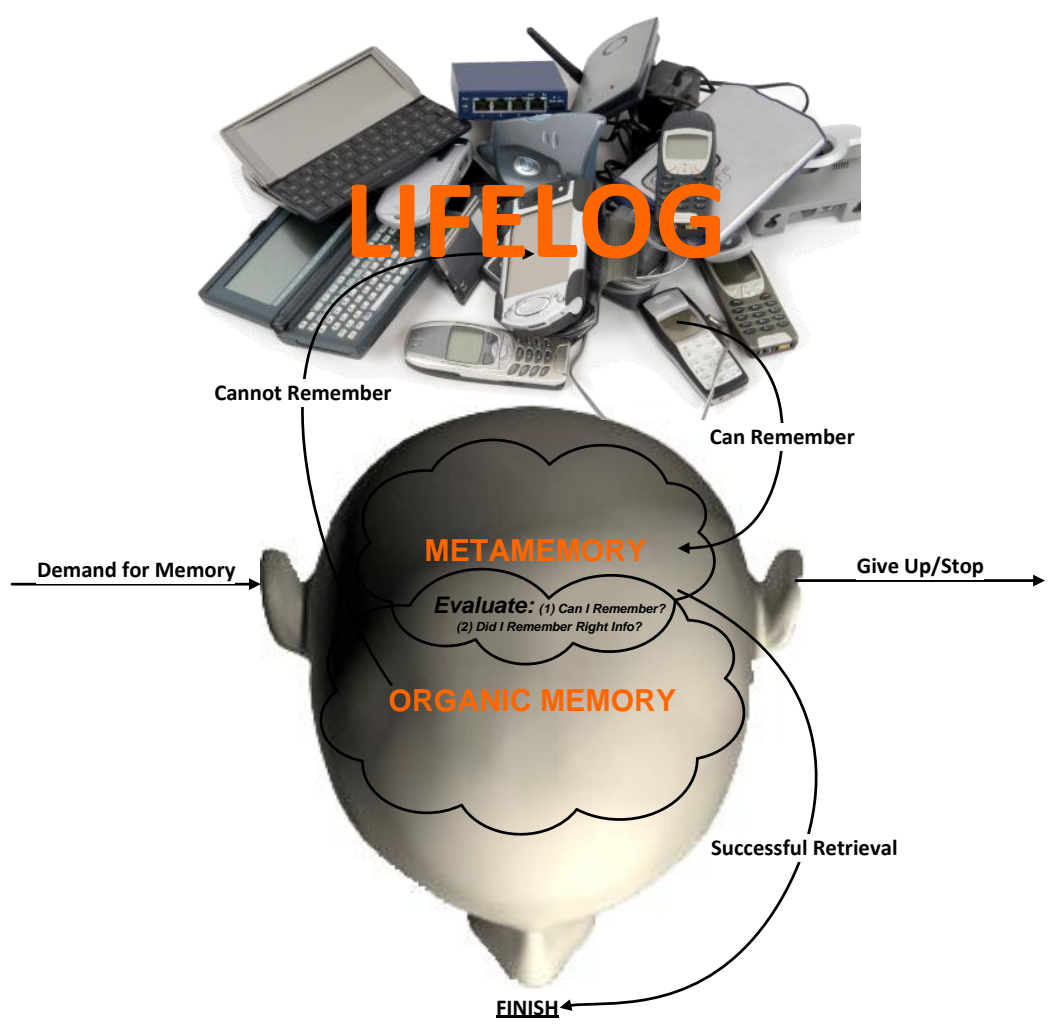

Figure 2: Interaction between metamemory and lifelogging tools during recollection.

Based on these continuous interactions between organic memory and Lifelog mediated by metamemory we derived a set of trade-offs that apply to different styles and motivations of remembering, see Table 1.

\begin{tabular}{|l|l|l|l|}
\hline \multicolumn{1}{|c|}{ Style of remembering } & \multicolumn{1}{|c|}{ Purpose } & \multicolumn{1}{c|}{ Motivation } & \multicolumn{1}{c|}{ Result } \\
\hline Organic remembering & Used for remembering gist & Efficiency & Loss of accuracy \\
\hline Synthetic remembering & $\begin{array}{l}\text { Used for accessing specific } \\
\text { information or for more } \\
\text { formal retrieval }\end{array}$ & $\begin{array}{l}\text { Influenced by failure to } \\
\text { remember organically }\end{array}$ & $\begin{array}{l}\text { Inefficient but more } \\
\text { accurate }\end{array}$ \\
\hline $\begin{array}{l}\text { Partial organic remembering } \\
\text { with reminders or lookup in } \\
\text { Lifelogs }\end{array}$ & $\begin{array}{l}\text { Used for quick reference or as } \\
\text { a reminder }\end{array}$ & $\begin{array}{l}\text { Influenced by failure to } \\
\text { remember organically }\end{array}$ & $\begin{array}{l}\text { Possibly efficient and } \\
\text { more accurate }\end{array}$ \\
\hline $\begin{array}{l}\text { Partial organic remembering } \\
\text { for gist together with a } \\
\text { Lifelog for detailed recall }\end{array}$ & $\begin{array}{l}\text { Used to cross-check the } \\
\text { accuracy of specific } \\
\text { information }\end{array}$ & $\begin{array}{l}\text { Demand for detailed } \\
\text { retrieval }\end{array}$ & $\begin{array}{l}\text { Possibly efficient and } \\
\text { more accurate }\end{array}$ \\
\hline
\end{tabular}

Table 1: Motivation for different types of recollection.

Empirical work suggest that lifelogging tools do indeed work in synergy with organic memory (Kalnikaite and Whittaker 2007), suggesting that metamemory processes are indeed active recollection from a Lifelog. But there are other motivations, e.g. speed of retrieval vs accuracy of retrieved information. Studies suggest that people overall prefer fast, but less accurate recollective methods, but these methods have to work in synergy with the way that 
their organic memories are cued. For instance, if the memory cue is generated organically, the lifelogging tool should really support that type of cue. Current lifelogging tools lack this flexibility and are unable to adapt to the changing nature of memory cuing tactics. Most lifelogging tools take the quickest approach of providing temporal or some other insignificant landmark related cues e.g. location or street name.

People generally are very good at evaluating their memories, in particular, what they know and what they do not know. People are also very aware of the fallibility of their organic memory which leads them to make strategic decisions on capturing important information on lifelogging devices.

When actively capturing information on Lifelogs it is important to dedicate enough attention in deciding what information should be captured and to process this information in advance. Previous research has shown that exhaustive capture of information does not necessarily aid retrieval (Kalnikaite and Whittaker 2007) - people prefer an efficient rather than an accurate record of the past. Other studies have shown that unintentional lifelogging capture can help retrieve information better than intentional capture (Sellen, Fogg et al. 2007). There are also trade-offs between different types of cues. In particular, visual and spatial (Kalnikaite, Sellen et al 2010) - visual cues provide detailed recall unlike spatial cues where recollection focus is on providing an overview of a day or an event.

There are ongoing discussions as to what retrieval cues are most effective, whether it is people, places, time or other (Cohen 1996). It is clear, however, that some context is usually required to create a meaningful cue to aid retrieval (Whittaker, Hirshberg et al. 2002; DeVaul, Pentland et al. 2003; Gemmell, Bell et al. 2006; Hodges, Williams et al. 2006). Research in material culture suggests that physical mementos can be effective memory cues too (Frohlich and Murphy 2000; Stevens, Abowd et al. 2003; Kawamura, Fukuhara et al. 2007).

The synergy between organic and synthetic remembering needs further research through application of metamemory research to inform future design of Lifelogging tools. Psychology research for decades has investigated how people remember and forget and this knowledge has not been exploited in the design of effective lifelogging tools. So far we have shown that people no longer rely solely on remembering organically, but they rely on metamemory to help them recollect the right information from their external, prosthetic Lifelogs. Our memories undergo immense challenges on a daily basis in terms of the amounts of information that are expected to be recalled. There are examples not only in the most memory demanding areas such as learning, but access to other areas of our everyday lives, including autobiographical recollection.

\section{Design Principles for Lifelogging Tools}

How can we design lifelogging tools that are relative, useful and can seamlessly fit into the fabric of our busy everyday lives? Psychology research provides insightful results into the human remembering processes, but it is still relatively rarely consulted when designing lifelogging tools. Designers of future lifelogging tools may benefit from taking into account key findings from the psychology literature on memory cues and, more generally, the process of remembering. In this section, we attempt to synthesize some of those findings into more readily applicable design patterns. 


\section{Synergy not substitution}

We have discussed the strong relationship between organic and synthetic remembering processes, which are proactively driven by metamemory. We suggest that people are only likely to use a lifelogging tool for recollection if they know that they have forgotten something they want to remember, and that this is the right tool to use to recall that memory. This has important implications for lifelogging approaches that focus on exhaustive capture of all experience, rather than cuing areas where users may have systematically poor organic recollection. Empirical research has shown that lifelogs are not a replacement for the actual process of organic remembering, and there is evidence that people may simply to supplement what they already remember, instead of consulting an entire recording of an event. For example, when using the ChittyChatty tool, people navigate through their hand written notes to listen to detailed recordings of the events rather than re-listening to an entire event (Kalnikaite and Whittaker 2010).

\section{Effective cueing}

Certain cues - such as people or life chapters - are more effective than temporal or location specific cues (Conway 2000, Cohen 1996). An appropriate design pattern should support fine-tuned memory cuing, including socially generated cues. Prevailing trends show personal and micro-blogging platforms - such as Facebook and Twitter - becoming part of many people's daily activity. Through these sites, people actively engage in self-documenting and lifelogging, creating, aggregating and sharing archives of self-projection. These platforms present rich opportunities for developing tools that explore techniques for effectively cuing memory and supporting self-reflection. One initial example in this direction is the Pensieve tool, which helps people reminisce by sending them occasional emails containing "memory triggers" (Peesapati et al 2010).

\section{Supporting memory where it is particularly weak}

"Stuff I have" tools help capture almost all types of personal memories. However, it is very unlikely that these vast repositories will be re-accessed again. Lifelogging tools should focus on ensuring the capture of only the most important or significant data through use of real time sensing technologies allowing users to decide on their memory weakness based on individual circumstance. These kinds of tools may be particularly well suited to support people with memory impairments, such as Alzheimer's patients.

\section{Instant feedback}

Many lifelogging tools take the 'black box' approach. The SenseCam device is one such example which ignores the benefits of instant feedback and leaves lifeloggers to speculate on what they have actually captured, and whether this is really what they wanted. Lack of instant feedback can lead to uncertainty in captured data, surfacing issues of distrust and personal privacy.

Seeing the alternate angle of life

Visual lifelogs often take the form of a stream of images captured from the viewpoint of an individual, and often exclude the lifelogger from appearing in their own lifelog. However, psychology research suggests that people tend to use self-referencing when remembering their past (Rubin 1988, Conway 2000). Thus designs for visual lifeloging tools may benefit 
from supporting third-person capture, allowing the lifelogger to see themselves from an external perspective.

\section{Conclusions}

Lifelogging research to date has been focused around enabling 'total capture', but psychology research suggests that lifelogging tools may benefit from being more selective. Instead of capturing everything, lifelogging tools should help users preserve the key events of the past or at least appropriately index and reference the key events in a lifelog. The recollective nature of memory should also be supported in lifelogging practices by building links between cues, and not necessarily focus on total recall. Empirical research suggests that we are very good at knowing what we can remember and what is likely to be forgotten, thus we can effectively prepare for this with the right type of lifelogging tool. This self-aware memory has been carefully tuned by the process of metamemory, which not only drives organic remembering, but it is also the key drive behind synthetic remembering.

Lifelogging tools need to work in synergy with organic remembering processes for effective cuing of past events. Rather than aiming to replace organic memory processes, future lifelogging tools should focus on designs that support this synergy rather than substitution. We should also design lifelogging to support memory where it is particularly weak. Future tools should integrate seamlessly into areas where people are generally prone to forgetting (e.g., by supporting prospective memory), or by designing specifically for populations that suffer from memory deficit, such as Alzheimer's (Berry, Kapur et al 2006). However, it is important to reemphasize that people tend to prefer tools that are efficient but not necessarily very accurate (Kalnikaite and Whittaker 2007).

Finally, "Things I Did" tools help us focus on the specific events from our past while "Stuff I Have" tools support aggregate information from multiple sources. The potential for these tools will be magnified by the increased availability of connected mobile devices, equipped with position and environmental sensors, cameras and audio recording capabilities, mass storage and online connectivity. These devices are already changing the way people capture, archive, self-document, share and socially recollect their surroundings and everyday experiences. These devices provide an ideal platform on which to develop and investigate new lifelogging tools that help us remember the things that we want - and know what we want - to remember.

\section{Bibliography}

Aizawa, K., D. Tancharoen, et al. (2004). Efficient retrieval of life log based on context and content. Proceedings of the the 1st ACM workshop on Continuous archival and retrieval of personal experiences. New York, New York, USA, ACM.

Anderson, R., R. Anderson, et al. (2004). Experiences with a tablet PC based lecture presentation system in computer science courses. Proceedings of the 35th SIGCSE technical symposium on Computer science education. Norfolk, Virginia, USA, ACM.

Bell, G. and Gemmell, J., (2009) Total Recall: How the E-Memory Revolution Will Change Everything. Dutton, New York. 
Berque, D., T. Bonebright, et al. (2004). Using pen-based computers across the computer science curriculum, ACM. 36: 61-65.

Berque, D., D. Johnson, K., et al. (2001). Teaching theory of computation using pen-based computers and an electronic whiteboard, ACM. 33: 169-172.

Berry, E., N. Kapur, et al. (2006). The use of a wearable camera, SenseCam, as a pictorial diary to improve autobiographical memory in a patient with limbic encephalitis.

Neuropsychological Rehabilitation.

Brewer, W. and C. Sampaio (2006). Processes leading to confidence and accuracy in sentence recognition: A metamemory approach. Memory 14: 540-552.

Brotherton, J. A. and G. D. Abowd (2004). Lessons learned from eClass: Assessing automated capture and access in the classroom, ACM. 11: 121-155.

Bush, V. (1945). As we may think. The Atlantic Monthly. 1: 101--108.

Cavanaugh, J. C. (1988). The place of awareness in memory development across adulthood. Everyday cognition in adulthood and later life, Cambridge University Press.

Cohen, G. (1996). Memory in the Real World, The Open University, Psychology Press.

Cohen, G. (1998). The effects aging on autobiographical memory. Autobiographical memory: theoretical and applied perspectives. C. P. Thomspson, D. J. Herrmann, D. Bruceet al. NJ, NJ: Lawrence Erlbaum Associates Inc: 105-123.

Cohen, G., G. Kiss, et al. (1993). Memory: Current Issues (Open Guide to Psychology), Open University Press.

Conway, M. A. and C. W. Pleydell Pearce (2000). The construction of autobiographical memories in the self memory system. Psychological Review(107): 261-288.

Cutrell, E., D. Robbins, et al. (2006). Fast, flexible filtering with phlat. Proceedings of the SIGCHI conference on Human Factors in computing systems. Montréal, Canada, ACM.

Davis, R. C., J. A. Landay, et al. (1999). NotePals: lightweight note sharing by the group, for the group. Proceedings of the SIGCHI conference on Human factors in computing systems: the $\mathrm{CHI}$ is the limit. Pittsburgh, Pennsylvania, United States, ACM.

DeVaul, R. W., S. A. Pentland, et al. (2003). The Memory Glasses: Subliminal vs. Overt Memory Support with Imperfect Information. Proceedings of the 7th IEEE International Symposium on Wearable Computers, IEEE Computer Society.

Dickie, C., R. Vertegaal, et al. (2004). Augmenting and sharing memory with eyeBlog. Proceedings of the the 1st ACM workshop on Continuous archival and retrieval of personal experiences. New York, New York, USA, ACM. 
Doherty, Aiden R. (2009) Providing effective memory retrieval cues through automatic structuring and augmentation of a lifelog of images. PhD thesis, Dublin City University.

Dourish, P., W. K. Edwards, et al. (1999). Presto: An Experimental Architecture for Fluid Interactive Document Spaces. ACM Transactions on Computer-Human Interaction 6(2): 133-161.

Dumais, S., E. Cutrell, et al. (2003). Stuff I've seen: a system for personal information retrieval and re-use. Proceedings of the 26th annual international ACM SIGIR conference on Research and development in informaion retrieval. Toronto, Canada, ACM.

Freeman, E. and D. Gelernter (1996). Lifestreams: a storage model for personal data, ACM. 25: 80-86.

Frohlich, D. and R. Murphy (2000). The Memory Box, Springer-Verlag. 4: 238-240.

Gemmell, J., G. Bell, et al. (2006). MyLifeBits: a personal database for everything. 49(1): 8895.

Harper, R., D. Randall, et al. (2008). The past is a different place: they do things differently there. Proceedings of the 7th ACM conference on Designing interactive systems. Cape Town, South Africa, ACM.

Harvel, L. D., C. Scheibe, et al. (2005). Technology design for connecting student notes to online course content. INTERACT.

Hayes, G., S. N. Patel, et al. (2004). The Personal Audio Loop: Designing a Ubiquitous Audio-Based Memory Aid. Mobile HCI 2004.

Healey, J. and W. R. Picard (1998). StartleCam: A Cybernetic Wearable Camera. Proceedings of the 2nd IEEE International Symposium on Wearable Computers, IEEE Computer Society.

Hodges, S., L. Williams, et al. (2006). SenseCam: A Retrospective Memory Aid. UbiComp 2006: Ubiquitous Computing: 177-193.

Johnson-Laird, P. N. (1988). The Computer and the Mind, Harvard University Press, Cambridge Mass.

Kalnikaite, V. and S. Whittaker (2007). Software or Wetware? Discovering When and Why People use Digital Prosthetic Memory. Proceedings of the SIGCHI conference on Human factors in computing systems. San Jose, California, USA, ACM Press.

Kalnikaité, V. and S. Whittaker (2008). Social Summarization: Does Social Feedback Improve Access to Speech Data? CSCW, San Diego, USA, ACM Press.

Kalnikaite, V. and Whittaker, S. (2010). A Saunter Down Memory Lane: Digital Reflections on Personal Mementos. International Journal of Human-Computer Studies. 
Kalnikaitè, V. and Whittaker S. (2010). Beyond Being There? Evaluating Augmented Digital Records. International Journal of Human-Computer Studies, 68(10) pp. 627-640.

Karger, D. R. and D. Quan (2004). Haystack: a user interface for creating, browsing, and organizing arbitrary semistructured information. CHI '04 extended abstracts on Human factors in computing systems. Vienna, Austria, ACM.

Kawamura, T., T. Fukuhara, et al. (2007). Ubiquitous Memories: a memory externalization system using physical objects. Personal and Ubiquitous Computing 11(4): 287-298.

Koriat, A. (2000). The feeling of knowing: Some metatheoretical implications for consciousness and control. Consciousness and congnition 9: 149-171.

Lachman, J. L., R. Lachman, et al. (1979). Metamemory through the adult life span. Developmental Psychology 15: 543-551.

Lamming, M. and M. Flynn (1994). "Forget-me-not" Intimate Computing in Support of Human Memory. FRIEND21, International Symposium on Next Generation Human Interface. Meguro Gajoen, Japan.

Linton, M. (1982). Transformation of memory in everyday life. Memory Observed: Remembering in natural context. U. E. Neisser. SF, San Francisco: Freeman.

Mann, S., J. Fung, et al. (2005). Designing Eye'Tap Digital Eyeglasses for Continuous Lifelong Capture and Sharing of Personal Experiences. Conference on Computer Human Interaction, ACM Press.

Munteanu, C., R. Baecker, et al. (2008). Collaborative editing for improved usefulness and usability of transcript-enhanced webcasts. Proceeding of the twenty-sixth annual SIGCHI conference on Human factors in computing systems. Florence, Italy, ACM.

Peesapati, S. T., Schwanda, V., Schultz, J., Lepage, M., Jeong, S., and Cosley, D. (2010). Pensieve: supporting everyday reminiscence. In Proceedings of the 28th international Conference on Human Factors in Computing Systems (Atlanta, Georgia, USA, April 10 - 15, 2010). CHI '10. ACM, New York, NY, 2027-2036.

Petrelli, D., Villar, N., Kalnikaitè, V., Dib, L., Whittaker, S. (2010) FMRadio: Family Interplay with Sonic Mementos. In Proceedings of the 28th international Conference on Human Factors in Computing Systems (Atlanta, Georgia, USA, April 10 - 15, 2010). CHI '10. ACM, New York, NY, 13-19.

Ratto, M., R. B. Shapiro, et al. (2003). The ActiveClass Project: Experiments in Encouraging Classroom Participation. CSCL.

Rekimoto, J. (1999). TimeScape: a time machine for the desktop environment. CHI '99 extended abstracts on Human factors in computing systems. Pittsburgh, Pennsylvania, ACM.

Rhodes, B. J. (2000). Margin notes: building a contextually aware associative memory. 
Proceedings of the 5th international conference on Intelligent user interfaces. New Orleans, Louisiana, United States, ACM.

Rosenschein, S. J. (2004). Quindi meeting companion: a personal meeting-capture tool. Proceedings of the the 1st ACM workshop on Continuous archival and retrieval of personal experiences. New York, New York, USA, ACM.

Rubin, C. D. (1988). Autobiographical Memory, Cambridge University Press.

Schacter, D. L. (1997). Searching For Memory: The Brain, The Mind, And The Past, Basic Books.

Sellen, A. J., A. Fogg, et al. (2007). Do life-logging technologies support memory for the past?: an experimental study using sensecam. Proceedings of the SIGCHI conference on Human factors in computing systems. San Jose, California, USA, ACM.

Stevens, M. M., G. D. Abowd, et al. (2003). Getting into the Living Memory Box: Family archives and holistic design. Personal and Ubiquitous Computing 7(3): 210-216.

Stifelman, L., B. Arons, et al. (2001). The audio notebook: paper and pen interaction with structured speech. Proceedings of the SIGCHI conference on Human factors in computing systems. Seattle, Washington, United States, ACM.

Tucker, S. and S. Whittaker (2006). Time is of the essence: an evaluation of temporal compression algorithms. Proceedings of the SIGCHI conference on Human Factors in computing systems. Montréal, Canada, ACM.

Vemuri, S., C. Schmandt, et al. (2006). iRemember: a personal, long-term memory prosthesis. Proceedings of the 3rd ACM workshop on Continuous archival and retrival of personal experences. Santa Barbara, California, USA, ACM.

Wellman, H. M. (1977). Tip of the tongue and feeling of knowing experiences: A developmental study of memory monitoring. Child Development, 48: 13-21.

Whittaker, S., J. Hirshberg, et al. (2002). SCANMail: a voicemail interface that makes speech browsable, readable and searchable. Proceedings of the SIGCHI conference on Human factors in computing systems: Changing our world, changing ourselves. Minneapolis, Minnesota, USA, ACM.

Whittaker, S., P. Hyland, et al. (1994). FILOCHAT: handwritten notes provide access to recorded conversations. Proceedings of the SIGCHI conference on Human factors in computing systems: celebrating interdependence. Boston, Massachusetts, United States, ACM.

Wilcox, D. L., N. B. Schilit, et al. (1997). Dynomite: a dynamically organized ink and audio notebook. Proceedings of the SIGCHI conference on Human factors in computing systems. Atlanta, Georgia, United States, ACM.

Wilkerson, M., G. W. Griswold, et al. (2005). Ubiquitous presenter: increasing student access 
and control in a digital lecturing environment, ACM. 